Article

\title{
Assessments of Population Structure, Diversity, and Phylogeography of the Swiss Needle Cast Fungus (Phaeocryptopus gaeumannii) in the U.S. Pacific Northwest
}

\author{
Patrick Bennett * and Jeffrey Stone \\ Received: 2 October 2015; Accepted: 17 December 2015; Published: 6 January 2016 \\ Academic Editors: Jan Stenlid, Jonas Oliva and Audrius Menkis \\ Department of Botany and Plant Pathology, Oregon State University, Corvallis, OR 97331, USA; \\ stonej@science.oregonstate.edu \\ * Correspondence: bennetpa@science.oregonstate.edu; Tel.: +1-541-737-8380
}

\begin{abstract}
Swiss needle cast (SNC) is a foliar disease of Douglas-fir (Pseudotsuga menziesii) caused by Phaeocryptopus gaeumannii (Rohde) Petrak. This fungus is endemic to western North America, where it has historically had little impact in native forests. However, increasing disease severity in western Oregon since the 1990s has prompted renewed interest in P. gaeumannii and SNC. For this study, we analyze multilocus microsatellite genotypes from 482 single-spore isolates from 68 trees across 14 sites in the western Coast Range of Oregon and southwestern Washington. This study assesses genotypic variation and genetic structure at several levels of population hierarchy. Despite the observation that most of the genetic variation occurred within subpopulations, our analyses detected significant differentiation at all hierarchical levels. Clustering among the 482 isolates based on genetic distance clearly supports the existence of two previously described cryptic lineages of P. gaeumannii in the western United States. The two lineages occur in varying proportions along latitudinal and longitudinal gradients in western Oregon and Washington, suggesting a relationship between climate and phylogeography. Sites near Tillamook, Oregon, where SNC is most severe, consist of sympatric subpopulations in which the two lineages comprise roughly equal proportions.
\end{abstract}

Keywords: Douglas-fir; forest pathology; population genetics; microsatellites; mycology

\section{Introduction}

Defoliation caused by Phaeocryptopus gaeumannii (Rohde) Petrak was first observed in Douglas-fir plantations in Europe in the 1920s and was termed "Swiss needle cast" (hereafter SNC). It was later discovered that this fungus is abundant and widespread in western North America, with herbarium specimens bearing P. gaeumannii pseudothecia collected as early as 1916 [1]. It was widely accepted that this fungus is ubiquitous throughout Douglas-fir forests in the U.S. Pacific Northwest, although it had rarely been observed to cause noticeable impacts outside of the Christmas tree industry [2]. Beginning in the mid-1980s symptoms of the disease began to emerge in the western Oregon Coast Range near Tillamook. Foresters noted chlorotic foliage, thinning crowns, and reduced height and diameter growth of Douglas-fir in timber plantations. Subsequent research identified P. gaeumannii as the most likely cause [2]. Later studies of SNC disease physiology revealed that these symptoms are caused by reduced gas exchange in the needles due to the occlusion of the stomata by ascocarps (pseudothecia) produced by this fungus. This blockage of the stomata leads to a reduction in carbon assimilation proportional to the percentage of total stomates occupied by pseudothecia [3] Abscission occurs when a needle ceases to function as a carbon source [4]. Regional SNC aerial surveys conducted annually since 1996 have documented gradual expansions in the area affected by the disease and in 
the magnitude of disease severity. By 2015, the total area in western Oregon displaying symptoms of SNC reached 237,250 ha, an increase of over 350\% since the first survey in 1996 [5].

Although P. gaeumannii reproduces sexually through the formation of ascospores in the annual production of pseudothecia; no form of asexual sporulation has been observed [6]. Despite this absence of true clonal reproduction, previous population genetic studies that employed molecular techniques such as SSCP (single strand conformation polymorphisms) suggested that a clonal population structure predominates, although outcrossing likely occurs with low frequency [7]. Given that the vegetative thallus of this fungus contains haploid nuclei, these observations likely reflect the role of homothallism in P. gaeumannii populations. In addition to this population substructure due to reproductive mode, the presence of multiple lineages of $P$. gaeumannii in the United States was demonstrated through the use of multilocus gene-genealogies [7]. An analysis of the spatial distributions of these lineages suggested that they co-occur in sympatric populations in the western United States, particularly in the western Coast Range in Oregon. In a sampling of five plantations in the SNC epidemic area, an increasing proportion of one of these lineages, "Lineage 2", was negatively correlated with foliage retention, a common measure of disease severity [8]. This lineage was also twice as likely to be recovered from sites that were rated as having severe SNC infections than those with less severe disease [8]. This observation led these authors to suggest that Lineage 2 might be more aggressive than Lineage 1 , and that its recent emergence may have been a significant factor in the intensification of SNC in northwestern Oregon. In a later analysis, 10 nuclear microsatellite loci were used to assess diversity and population structure in a sample of 60 isolates from western Oregon [9]. The authors noted that genotypic diversity was exceptionally high in their sample population. High diversity, combined with the apparent lack of linkage disequilibrium between many of the loci, led these authors to conclude that this particular set of microsatellite markers should serve as an appropriate tool for the estimation of population diversity and structure for P. gaeumannii. They also noted that sampling at finer spatial scales was needed to accurately assess these parameters and to determine the relationship between lineage phylogeography and the distribution of disease on the landscape [9].

The objectives of this study were to analyze the distributions of the two lineages of P. gaeumannii at multiple spatial scales across geographic and climatic gradients. Estimates of genotypic diversity and population differentiation were considered in an attempt to correlate population dynamics with possible factors influencing the observed distribution of disease severity in western Oregon. Our analyses aimed to assess the extent to which gene flow occurs between subpopulations at several levels of a population hierarchy. Lineages, sampling sites, and individual trees were considered as possible demes in which random mating may occur. This study also aims to assess the spatial scales at which populations of $P$. gaeumannii are structured in natural populations. To address this question, samples were collected and analyzed from several spatial scales ranging from trees to landscapes. Genetic clustering analyses were performed in which the similarity and relatedness between isolates from various spatial scales could be compared statistically as well as graphically.

\section{Materials and Methods}

\subsection{Sample Collection}

Included in these analyses are microsatellite genotypes from 482 isolates of P. gaeumannii collected from 68 trees in 14 Douglas-fir plantations in western Oregon and southwestern Washington. Nine sites were sampled in the Coast Range in northern Oregon near the town of Tillamook, with a central transect of five sites spanning $57 \mathrm{~km}$ west-to-east. Three sites were sampled in the central Oregon Coast Range spanning 24-57 km east of the town of Florence, and two sites were sampled approximately $40 \mathrm{~km}$ inland in southwestern Washington (Figure 1, Table 1). Foliage was collected from five randomly selected 10-30 year old Douglas-fir trees at each site. Second- and third-year internodes were sampled from secondary branches in the upper crown. From one tree at each site, branches were also collected from vertically stratified sections of the canopy including the lower, middle, and upper crown 
(Figure 1B). The number of isolates obtained from each tree ranged from 10 to 30, and the number of isolates genotyped from each sampling site ranged from 10 to 99 .

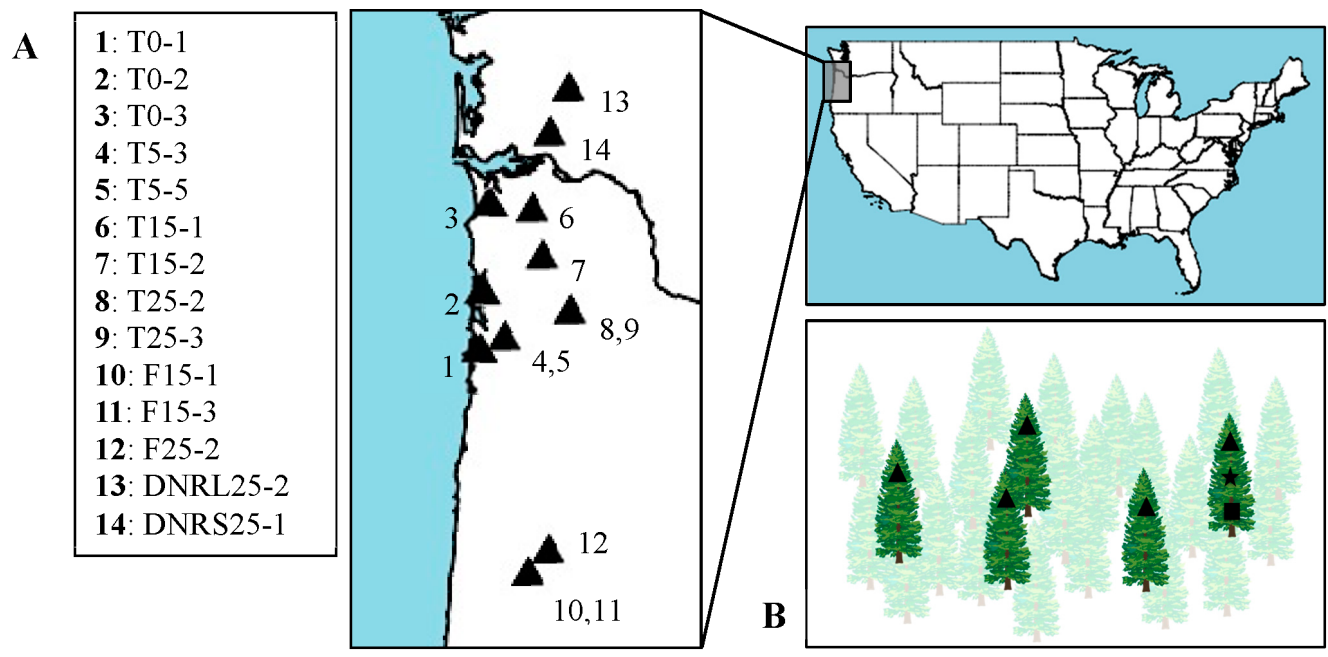

Figure 1. (A) Map of northwestern Oregon and southwestern Washington with markers indicating the locations of sites where Pseudotsuga menziesii foliage was collected for isolation of Phaeocryptopus gaeumannii. Legend at left shows the site names corresponding to marker numbers on map. $\mathrm{T}=$ Tillamook, $\mathrm{F}=$ Florence, $\mathrm{DNR}=$ Washington Department of Natural Resources. The number immediately following the letter corresponding to these locations represents the distance of the sampled stand from the coast. (ex: T25 $=25-35$ miles $(40-57 \mathrm{~km})$ from coast at the latitude of Tillamook, Oregon); (B) Schematic diagram of foliage sampling at a given site. Triangles represent collections from the upper canopies of five randomly selected Douglas-fir trees at each site. The star and the square represent collections of foliage from the middle and lower canopy, respectively, of one of the five trees sampled at each site.

Table 1. Sample sizes, genotypic richness, and lineage distributions determined for 482 P. gaeumannii isolates from the 14 sites sampled for this study.

\begin{tabular}{cccccc}
\hline Site & $\mathbf{N}^{\mathbf{a}}$ & $\mathbf{M L G}^{\mathbf{b}}$ & $\mathbf{e M L G}^{\mathbf{c}}$ & $\mathbf{L 1}^{\mathbf{d}}$ & $\mathbf{L 2}^{\mathbf{e}}$ \\
\hline $\mathrm{T}-01$ & 49 & 48 & 9.96 & 23 & 26 \\
$\mathrm{~T}-02$ & 10 & 9 & 9 & 4 & 6 \\
T-03 & 17 & 17 & 10 & 9 & 8 \\
T5-3 & 29 & 29 & 10 & 22 & 7 \\
T5-5 & 21 & 20 & 9.79 & 17 & 4 \\
T15-1 & 46 & 45 & 9.96 & 23 & 23 \\
T15-2 & 24 & 23 & 9.84 & 10 & 14 \\
T25-2 & 99 & 90 & 9.85 & 99 & 0 \\
T25-3 & 60 & 59 & 9.97 & 59 & 1 \\
F15-1 & 29 & 27 & 9.78 & 27 & 2 \\
F15-3 & 15 & 15 & 10 & 4 & 11 \\
F25-2 & 37 & 32 & 9.61 & 25 & 12 \\
DNRL25-2 & 25 & 25 & 10 & 19 & 6 \\
DNRS25-2 & 21 & 16 & 8.93 & 13 & 8 \\
Total & 482 & 454 & 9.99 & 358 & 124 \\
\hline
\end{tabular}

a $\mathrm{N}=$ number of isolates; ${ }^{\mathrm{b}} \mathrm{MLG}=$ number of multilocus genotypes; ${ }^{\mathrm{c}}$ eMLG $=$ genotypic richness (expected number of multilocus genotypes in a rarefied sample of 10 isolates); ${ }^{\mathrm{d}} \mathrm{L} 1=$ abundance of Lineage 1; ${ }^{\mathrm{e}} \mathrm{L} 2=$ abundance of Lineage 2. 


\subsection{Isolation and Culturing}

Needles with mature pseudothecia of P. gaeumannii were selected for ascospore isolations. Groups of six needles from each sample were attached to the lids of $100 \mathrm{~mm}$ styrene Petri dishes with double-sided tape and suspended above the agar surface to allow ascospore discharge. After 48-72 h, individual germinating ascospores were excised from the agar surface with the aid of flame-sterilized forceps and isolated onto 2\% malt agar (MA) (Difco Laboratories, Detroit, MI, USA). Site information, tree number, and a unique isolate number were recorded for each spore. Cultures were incubated at $17^{\circ} \mathrm{C}$ for 2-6 months to allow sufficient growth for DNA extraction and permanent storage.

\subsection{DNA Extractions}

Total genomic DNA was extracted from vegetative mycelium using the DNeasy Plant Mini Kit 250 (Qiagen, Hilden, Germany). The protocol followed the manufacturer's instructions with the addition of an initial maceration procedure. Agar plugs extracted from P. gaeumannii cultures were added to cryogenic vials with sterile $2 \mathrm{~mm}$ glass beads. The vials were briefly submerged in liquid nitrogen to freeze the agar plugs prior to the addition of the DNeasy extraction buffer AP1. A Mini-Beadbeater-1 (BioSpec Products, Bartlesville, OK, USA) was then used to agitate each of the vials at $5000 \mathrm{rpm}$ for $60 \mathrm{~s}$.

\subsection{Microsatellite PCR}

For each isolate, ten microsatellite loci [9] were amplified in three multiplexed PCR reactions. The multiplexed reactions contained between two and five primer sets, each with a fluorescent dye label on the reverse primer. The sequences of the primers were identical to those described in [9] but without the $18 \mathrm{bp}$ M13 universal tails, resulting in shorter PCR products. The PCR reactions were performed using the Qiagen Type-It Microsatellite PCR kit, and the protocol was employed according to the manufacturer's instructions, but with reaction volumes of $12.5 \mu \mathrm{L}$. The amplification was performed with a PTC-200 thermal cycler (MJ Research, Inc. Waltham, MA, USA) programmed according to [9].

\subsection{Genotyping and Allele Scoring}

Each of the multiplexed PCR reactions was diluted by a factor of 10 in deionized water, and $1 \mu \mathrm{L}$ of the diluted product was submitted to the Center for Genome Research and Biocomputing (CGRB, Oregon State University, Corvallis, OR, USA) for genotyping via capillary electrophoresis on an ABI 3730 DNA Analyzer (Applied Biosystems-ThermoFisher Scientific Corporation, Waltham, MA, USA) with the GS-500ROX size standard. Allele sizes and genotypes were assigned with the aid of the ABI GeneMapper 4.0 software (Applied Biosystems-ThermoFisher Scientific Corporation) Bins were set such that samples could be identified based on their sizes and fluorescent labels, and panels were set to recognize previously reported allele size ranges [9] adjusted to account for the absence of the M13 primer tails. Microsatellite alleles were called using the standard algorithm in the GeneMapper software, and were also validated visually at each locus for each isolate.

\subsection{Data Analysis}

Multilocus genotypes from a total of 482 P. gaeumannii isolates were included in this study. Data formatting and analyses were performed with $\mathrm{R}$ version 3.2.1 [10] and GenAlEx 6.5 [11,12]. The formatted genotypes were imported to $\mathrm{R}$ for use with Poppr 2.0.2 [13]. For all analyses described here, the mutlilocus genotype data were stratified in a population hierarchy, as described in [14]. Lineages were considered as the most inclusive level of this hierarchy, sampling sites were considered subpopulations within lineages, and trees were considered subpopulations within sites.

Shannon-Weiner diversity (H), Stoddart and Taylor's index (G) [15], Simpson's Index $(\lambda)[16]$, expected heterozygosity $\left(\mathrm{H}_{\mathrm{e}}\right.$, Nei's gene diversity) [17], and genotypic richness, were compared at two levels of the population hierarchy, site and lineage. Genotypic richness was estimated as the 
number of expected multilocus genotypes (eMLG) in a rarefied sample size (n). These diversity parameters were chosen based on their descriptive power and utility as outlined by [18]. Because these analyses are sensitive to differences in sample sizes between the populations, diversity was assessed on rarefied sub-samples of the data. For a comparison of diversity between lineages, 128 isolates were included in the analysis. For a comparison of diversity across sites, the sample sizes were rarefied to 10 isolates.

Analysis of molecular variance (AMOVA) was used to compare the genetic variation within and between the levels of the population hierarchy. Estimates of $\phi$ were used to infer gene flow and population differentiation [19]. For these analyses, clone-correction was used to remove repeated MLGs. Statistical significance was assessed with randomization tests with 999 permutations.

The unweighted pair group method with arithmetic means (UPGMA) algorithm was used to construct dendrograms. Their associated bootstrap statistics were calculated using Poppr 2.0.2 and dendrograms were visualized with FigTree version 1.4.2 [20]. UPGMA method groups organisms together based on genetic distance determined by the similarity of their multilocus genotypes. An analysis of branching patterns and clustering allowed for the inference of population structure. For the assessments of population structure and lineage membership, all of the 482 isolates were used to construct a bootstrapped UPGMA dendrogram. For analyses of genetic clustering among isolates at smaller spatial scales, dendrograms were constructed with random samples of 100 isolates from a clone-corrected data set. For bootstrapping, 1000 replicate trees were sampled. Rogers' distance [21], a Euclidean measure of genetic distance, was chosen for this analysis due to the fact that it does not rely upon assumptions that the microsatellite loci evolve by the stepwise mutation model.

Mantel's test, an estimate of spatial autocorrelation, was performed to test for correlations between geographic distance and genetic distance. A matrix of genetic distances between populations, calculated according to [21], was compared to a matrix of Euclidean distances between the geographic coordinates of each of the sampling sites. A randomization test was performed in which the data were permuted 999 times to obtain a $p$-value.

\section{Results and Discussion}

\subsection{Richness and Diversity}

All 10 of the microsatellite loci employed in this study were polymorphic. The number of alleles at each locus ranged from 13 to 53 . Of the 482 isolates included in the analyses, a total of 454 unique MLGs were identified (Table 1). A total of 354 multilocus genotypes (MLGs) were consistent with those previously described as Lineage 1, and 128 were consistent with previous descriptions of Lineage 2 [9]. Genotypic diversity indices, including Shannon-Wiener, Stoddart and Taylor's, and Simpson's, were significantly greater for Lineage 1 than Lineage 2 (Table 2). Comparisons of genotypic diversity in rarefied samples of 10 isolates from each site revealed a general geographic trend of diversity increasing from west to east at the latitude of Tillamook, Oregon (Figure 2A). The site with the least genotypic diversity was the westernmost site, T- 02 , while the greatest occurred at site T25-2, approximately $50 \mathrm{~km}$ east of T-02. This increase in genetic diversity coincided with an increasing proportion of Lineage 1 isolates in sites east of Tillamook (Table 1, Figures 2A and 3). The high genetic diversity within Lineage 1 suggests that it is native to the eastern Coast Range, where it is most abundant and diverse, as suggested in [8]. A comparison of genotypic diversity between 10 isolates of each lineage revealed that Lineage 1 is significantly more diverse than Lineage 2 (Table 2, Figure 2B). 


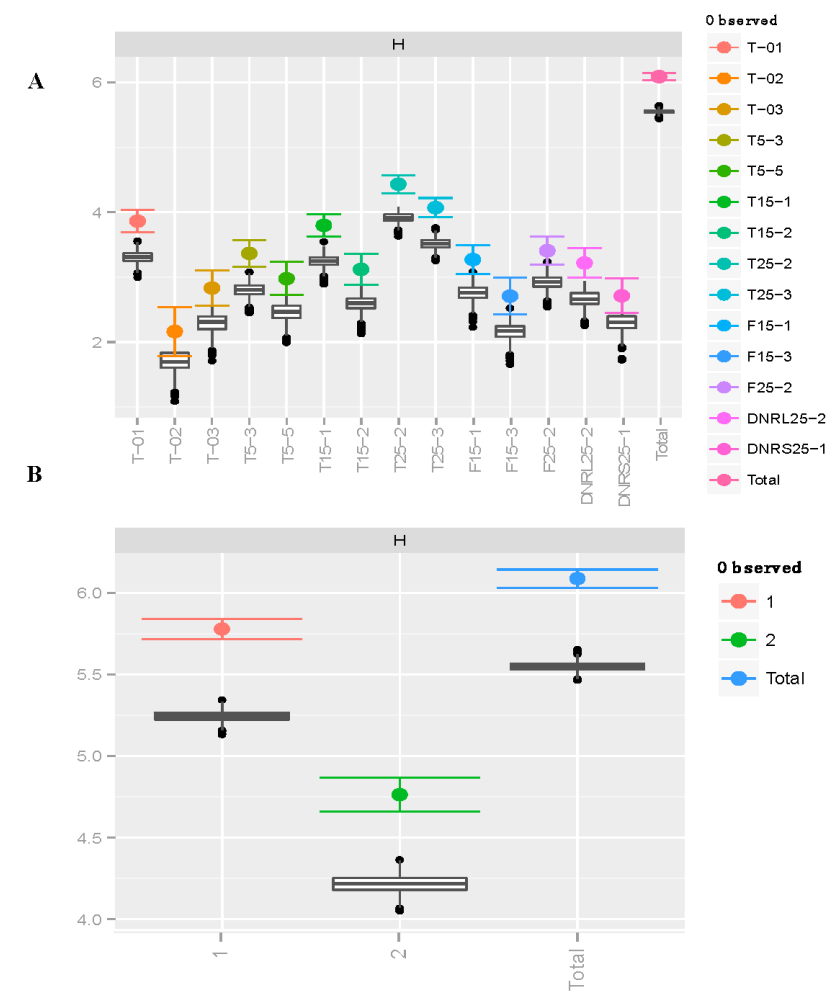

Figure 2. (A) Comparisons of P. gaeumannii genotypic diversity by sampling site. $N=10$; (B) Comparisons of P. gaeumannii genotypic diversity by lineage. $1=$ Lineage 1, $2=$ Lineage $2 . N=128$. $\mathrm{H}=$ Shannon-Weiner diversity. The colored dots with error bars represent the observed statistics and their associated $95 \%$ confidence intervals. The black and white box and whisker plots represent the values estimated via bootstrapping with 1000 replicates.

Table 2. Diversity and heterozygosity of multilocus P. gaeumannii genotypes from two levels of the population hierarchy. (A) Diversity and heterozygosity measured for each sampling site ( $N=10$ isolates). (B) Diversity and heterozygosity values for each lineage ( $N=128$ isolates).

\begin{tabular}{|c|c|c|c|c|}
\hline \multicolumn{5}{|l|}{ A } \\
\hline Site & $\mathrm{H}^{\mathrm{a}}$ & $\mathrm{G}^{\mathrm{b}}$ & $\lambda^{c}$ & $\mathrm{H}_{\mathrm{e}}{ }^{\mathrm{d}}$ \\
\hline $\mathrm{T}-01$ & $3.864(3.69,4.04)$ & $47.08(42.0,52.1)$ & $0.979(0.97,0.99)$ & 0.821 \\
\hline $\mathrm{T}-02$ & $2.164(1.79,2.54)$ & $8.333(6.30,10.37)$ & $0.880(0.79,0.97)$ & 0.749 \\
\hline $\mathrm{T}-03$ & $2.833(2.56,3.11)$ & $17.00(14.3,19.8)$ & $0.941(0.90,0.98)$ & 0.806 \\
\hline T5-3 & $3.367(3.16,3.57)$ & $29.00(25.5,32.5)$ & $0.966(0.95,0.98)$ & 0.781 \\
\hline T5-5 & $2.979(2.72,3.23)$ & $19.17(16.0,22.3)$ & $0.948(0.92,0.98)$ & 0.726 \\
\hline T15-1 & $3.799(3.62,3.97)$ & $44.08(39.3,48.9)$ & $0.977(0.97,0.99)$ & 0.778 \\
\hline $\mathrm{T} 15-2$ & $3.120(2.88,3.36)$ & $22.15(18.8,25.6)$ & $0.955(0.93,0.98)$ & 0.804 \\
\hline $\mathrm{T} 25-2$ & $4.433(4.30,4.57)$ & $73.69(65.1,82.3)$ & $0.986(0.98,0.99)$ & 0.630 \\
\hline $\mathrm{T} 25-3$ & $4.071(3.92,4.22)$ & $58.07(52.8,63.3)$ & $0.983(0.98,0.99)$ & 0.717 \\
\hline F15-1 & $3.272(3.05,3.50)$ & $25.49(21.8,29.2)$ & $0.961(0.94,0.98)$ & 0.751 \\
\hline F15-3 & $2.708(2.43,2.99)$ & $15.00(12.5,17.5)$ & $0.933(0.89,0.98)$ & 0.810 \\
\hline F25-2 & $3.409(3.20,3.62)$ & $27.94(23.6,32.3)$ & $0.964(0.95,0.98)$ & 0.815 \\
\hline DNRL25-2 & $3.219(2.99,3.45)$ & $25.00(21.7,28.3)$ & $0.960(0.94,0.98)$ & 0.787 \\
\hline DNRS25-1 & $2.714(2.45,2.98)$ & $14.23(11.5,17.0)$ & $0.930(0.89,0.97)$ & 0.759 \\
\hline Total & $6.089(6.03,6.14)$ & $417.8(401,435)$ & $0.998(0.997,0.998)$ & 0.820 \\
\hline \multicolumn{5}{|l|}{ B } \\
\hline Lineage & $\mathbf{H}$ & G & $\lambda$ & $\mathbf{H}_{\mathrm{e}}$ \\
\hline 1 & $5.780(5.71,5.85)$ & $303.7(289,318)$ & $0.997(0.996,0.997)$ & 0.728 \\
\hline 2 & $4.764(4.66,4.87)$ & $114.7(107,122)$ & $0.991(0.989,0.993)$ & 0.745 \\
\hline Total & $6.089(6.03,6.15)$ & $417.8(400,435)$ & $0.998(0.997,0.998)$ & 0.820 \\
\hline
\end{tabular}

${ }^{a} \mathrm{H}=$ Shannon-Weiner diversity index; ${ }^{\mathrm{b}} \mathrm{G}=$ Stoddart and Taylor's index; ${ }^{\mathrm{c}} \lambda=$ Simpson's index; ${ }^{\mathrm{d}} \mathrm{H}_{\mathrm{e}}=$ Nei's expected heterozygosity. Numbers in parentheses are $95 \%$ confidence intervals calculated by bootstrapping. 


\subsection{Phylogeography}

The two lineages were recovered in varying proportions across the 14 study sites (Figure 3). Lineage 1 was recovered from all sites and comprised $26 \%-100 \%$ of total isolates at any given site; Lineage 2 comprised $0 \%-74 \%$ of isolates at the site level (Table 1). Lineage 2 was most abundant at sites within $16 \mathrm{~km}$ inland near Tillamook, Oregon, with decreasing abundance along this latitudinal transect to the east. All but one of the 158 isolates analyzed from sites T25-2 and T25-3 ( 50 km inland from Tillamook, OR, USA) were determined to belong to Lineage 1 (Figure 3, Table 1). The same geographic trend did not occur further south, as the F25-2 site ( $50 \mathrm{~km}$ inland from Florence, OR, USA) comprised approximately $32 \%$ Lineage 2 . The two sites in southwestern Washington, DNRL25-2 and DNRS25-2 ( 50 km inland), comprised $24 \%$ and $21 \%$ Lineage 2 , respectively.

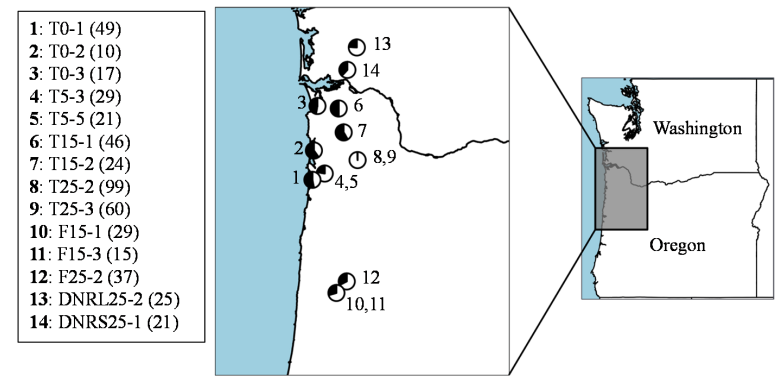

Figure 3. Map showing the relative distributions of two lineages of P. gaeumannii across the 14 sites sampled for this study. Lineage 1 is represented by empty (white) circles, while Lineage 2 is represented by filled (black) circles. The numbers next to each pie chart correspond to the site names in the legend at left, and the numbers in parentheses indicate sample sizes. Lineage proportions from sites 4 and 5, 8 and 9, 10 and 11, respectively, were pooled and displayed as single pie charts to avoid overlap.

The primary focus of this investigation was to correlate lineage phylogeography with disease distribution. In this case, climate (i.e., temperature and precipitation gradients) may be an important factor influencing the different distribution patterns of the two lineages across the landscape. There is strong evidence that climatic variables such as winter temperature and precipitation in the spring and early summer influence the abundance of P. gaeumannii, and thus disease [22-26]. The western Oregon Coast Range is particularly suited to support abundant populations of $P$. gaeumannii because it receives greater spring precipitation, and has generally milder winter, and cooler summer, temperatures than the rest of the region. The observed distribution of the lineages observed in this study and others [8,22], may be strongly influenced by seasonal temperatures. Trends in phylogeography also suggest that environmental conditions may limit the distribution of Lineage 2 relative to Lineage 1. One explanation may be that Lineage 2 is better adapted to warmer winter temperatures and thus is limited by colder winter temperatures to the east. Conditions in the northern Oregon Coast Range may have become particularly suitable for the growth and proliferation of P. gaeumannii and SNC in recent decades due to the effects of climate change [22,24] as well as forest practices favoring increased abundance of Douglas-fir relative to other species [2]. Both favorable environmental conditions and abundance of a susceptible host may have contributed to a recent expansion or migration of Lineage 2 into this region from coastal areas in southern Oregon where it appears to be more dominant.

It is important to note that there does not appear to be a particularly high incidence or severity of SNC at sites near the southern coast of Oregon [8] where Lineage 2 was most abundant in the analysis in [5]. Furthermore, where Lineage 1 dominates in Douglas-fir forests further inland, SNC severity is generally low or moderate. These trends, and the findings of the present study in which sites in the region known to have the greatest severity of SNC disease were found to have a combination of the two lineages, suggest that some synergistic effect or competitive interaction may be occurring between the two lineages. While the occurrence of hybridization between the two lineages may 
be possible (a lack of somatic compatibility has yet to be demonstrated) it is considered unlikely, given the strong differentiation and low rates of gene flow between the lineages found in this study. Phylogenetic studies that utilized nucleotide sequence data [7] also suggested that the lineages might be reproductively isolated from one another, thus making interbreeding and hybridization improbable. An alternative explanation for the relationship between disease severity and lineage coincidence may be that a recent emergence of more aggressive races of $P$. gaeumannii has occurred in this region. While this has been proposed as a potential explanation for the recent disease outbreak, direct assessments of aggressiveness among strains collected from sites varying in disease severity have proven inconclusive [8]

\subsection{Population Structure and Differentiation}

The existence of two lineages of P. gaeumannii was first posited by Winton [8] on the basis of SSCP genotypes. Their differentiation was also supported by mutlilocus gene genealogies of highly conserved nuclear housekeeping genes [7]. While it appears that the two lineages are distinct subpopulations that coexist in relatively close spatial proximity, reproductive isolation between them has yet to be definitively demonstrated. A comparison of genetic variation within and between two levels of the population hierarchy, lineage and site, revealed significant subpopulation differentiation. Most of the genetic variance in the total sample population could be attributed to within-tree variation (61.8\%, Table 3). Between-lineage variation accounted for $22.7 \%$ of the total, sampling site accounted for $4.7 \%$, and the remaining variation $(10.8 \%)$ occurred due to variation among trees within sites (Table 3). The $\phi$ statistic provides an estimate of population differentiation and gene flow among the various levels of the population hierarchy. Values of $\phi$ in the range of 0.15 to 0.25 reflect a high degree of differentiation, and are generally the result of low gene flow [27]. While the two lineages were highly differentiated $\left(\phi_{\mathrm{LT}}=0.227, p=0.001\right)$, sites were only slightly differentiated from one another within lineages $\left(\phi_{\mathrm{SL}}=0.061, p=0.001\right)$. Subpopulations of $P$. gaeumannii within individual trees also appear to be significantly differentiated from one another, suggesting a local establishment of infection from inoculum within the same tree $\left(\phi_{\mathrm{TS}}=0.148, p=0.001\right)$ (Table 3). The fact that lineages are highly differentiated suggests that they may be reproductively isolated due to behavioral differences or genetic incompatibilities. While $\phi_{\mathrm{LT}}$ theoretically should be close to 1 if there is complete reproductive isolation, it is quite rare to observe F-statistics or their analogs approaching 1, even among populations that are thought to be reproductively isolated [27]. Whether the observed within-lineage variance reflects the actual rate of gene flow and admixture, or is an artifact of a recent divergence of the lineages, is not entirely clear. Since microsatellites have very high rates of mutation, it is also possible that the apparent admixture between lineages may be due to the convergence of isolates from each lineage upon the same alleles at some microsatellite loci through random mutation (homoplasy).

When all samples were displayed on a UPGMA dendrogram based on Rogers'genetic distance [21] the basal branches split the 482 isolates into two discrete sub-population groupings that corresponded to previously identified lineages [9] (Figure 4). The divergence between these groups was statistically supported by a bootstrap value of $100 \%$ for 1000 replicate trees. The grouping of isolates was used to infer relatedness at various levels of the population hierarchy. For example, isolates from the same tree within a site clustered together with relatively high bootstrap support in many cases. In a UPGMA dendrogram produced from a random subsample of 100 individuals, single-spore isolates 5 and 6 , isolated from tree 701 at site T-03, clustered together in $99.5 \%$ of replicate trees (Figure 5A). On an adjacent branch, isolates from two different trees, 1744 and 1752 at site T5-5, clustered together in $69.4 \%$ of replicate trees (Figure $5 \mathrm{~B}$ ). There is little statistical support for genotypic similarity between isolates from trees at different sites. For example, isolates from sites that are 15 miles (24 km) apart, T-01 and T15-1, only clustered together in 53.7\% of replicate trees (Figure 5C). From the UPGMA analyses, it is clear that individuals from the same trees generally have very similar genotypes, suggesting a high level of relatedness and local dispersal. As the spatial distance between samples increases, the 
genetic similarity of corresponding isolates decreases. For instance, isolates that clustered together in the UPGMA analyses that came from the same trees had greater bootstrap support for their close genetic similarity than those that came from different trees within the same site, while there was little statistical support for clustering among isolates from more distant sites. This suggests that some degree of isolation by distance (IBD) exists, even though the Mantel test did not indicate a significant relationship between geographic distance and genetic distance $\left(R^{2}=0.088, p=0.271\right)$.

Table 3. Table summarizing the results of the analysis of molecular variance (AMOVA) performed with the 454 P. gaeumannii isolates possessing unique MLGs. The relative genetic variances explained by each of the levels of the population hierarchy are shown, and $\phi(P h i)$ statistics are shown as estimates of subpopulation differentiation and gene flow.

\begin{tabular}{cccc}
\hline Hierarchical Level & Variance (\%) & $\boldsymbol{\phi}$ & $\boldsymbol{p}^{*}$ \\
\hline$\phi_{\mathrm{LT}}$ (between lineages) & 22.671 & 0.227 & 0.001 \\
$\phi_{\mathrm{SL}}$ (among sites within lineages) & 4.719 & 0.061 & 0.001 \\
$\phi_{\mathrm{TS}}$ (among trees within sites) & 10.772 & 0.148 & 0.001 \\
$\phi_{\mathrm{TT}}$ (within trees) & 61.838 & 0.382 & 0.001 \\
\hline
\end{tabular}

* $p$-Value was obtained for the $\phi$ statistic using a randomization test with 999 permutations.

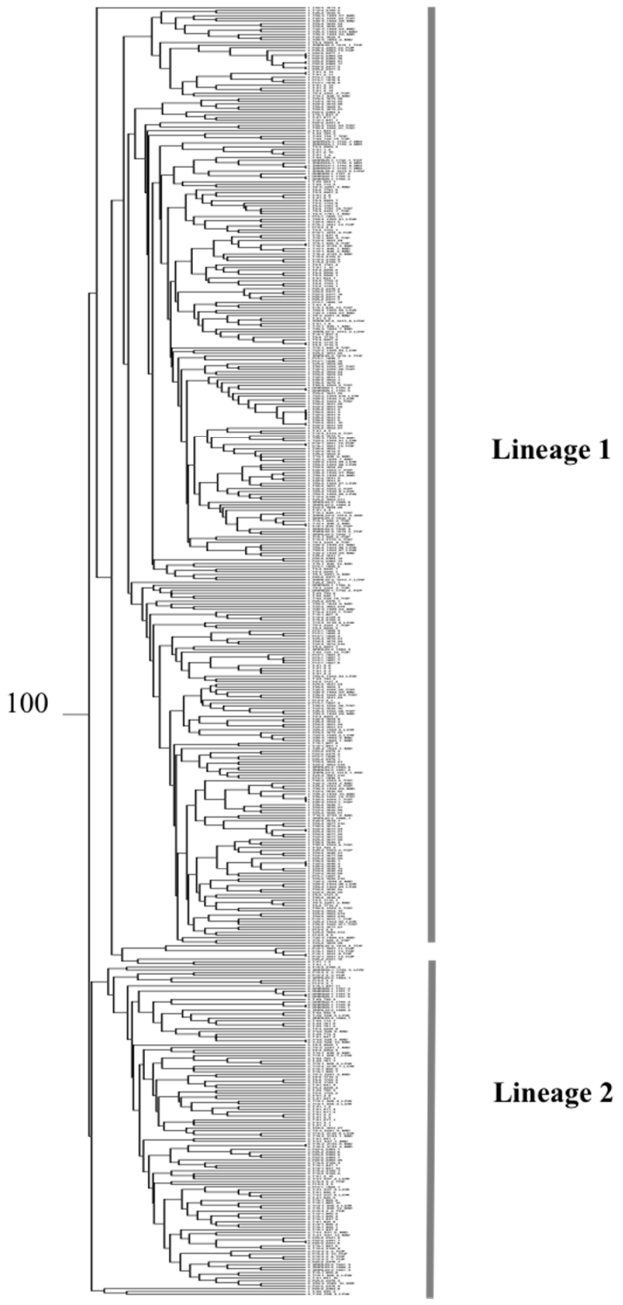

Figure 4. Unweighted pair-group method using arithmetic means (UPGMA) dendrogram showing clustering among the 482 P. gaeumannii isolates due to genotypic similarity. The basal node label represents the bootstrap statistic from 1000 replicate trees. Genetic distance was calculated according to Rogers (1972). 


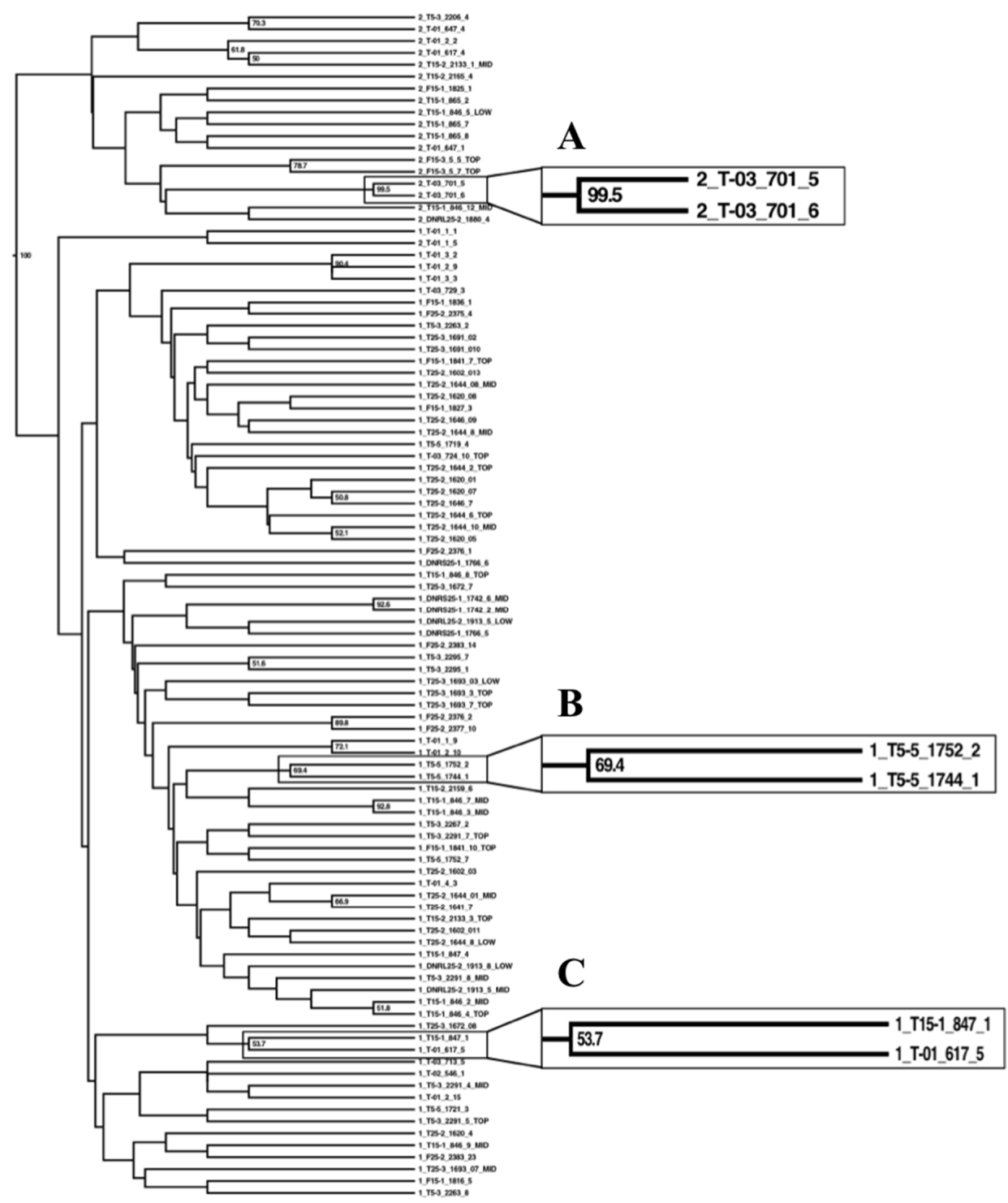

Figure 5. Unweighted pair-group method using arithmetic means (UPGMA) dendrogram constructed using 100 randomly selected P. gaeumannii isolates from the clone-censored data set. Node labels represent bootstrap statistics from 1000 replicate trees. Genetic distance was calculated according to Rogers (1972). (A) An example of clustering between two isolates from the same tree (701) at site T-03; (B) An example of clustering between two isolates from different trees $(1752,1744)$ at the same site (T5-5); (C) Clustering between two isolates from trees at two different sites (T15-1 and T-01).

\section{Conclusion}

While the relative aggressiveness of the two lineages is not known, the results of this study seem to support previous findings that suggest that $P$. gaeumannii lineage abundance and distribution is determined by winter temperature and precipitation. These findings are significant due to the fact that disease severity is determined primarily by pathogen abundance. The distribution of Lineage 2 is restricted to the western Coast Range in northern Oregon, and is virtually absent from Douglas-fir forests and plantations in the northeastern Coast Range. This distribution appears to be correlated with SNC disease severity assessed by annual aerial surveys. Sites where the two lineages occur in sympatric local populations are located within $50 \mathrm{~km}$ of the coast and exhibit the most severe SNC symptoms. The genetic structure of the P. gaeumannii populations included in this study supports reproductive isolation, as there is strong differentiation between lineages. Populations of this fungus 
are not structured geographically, and genetic differentiation among sites is weak suggesting high rates of gene flow. Information about the population structure of this fungus should be used to improve predictive models and management strategies used in the control of this disease and the mitigation of its impacts in Douglas-fir silviculture.

Future studies in this pathosystem should focus on direct assessments of aggressiveness among isolates from the two lineages, and also among isolates collected from sites with varying degrees of disease severity, through inoculation trials. It is also of interest to further pursue molecular and genomic studies to learn more about the phylogenetic relationship between the two lineages. Whether they could be considered separate species cannot be determined using markers such as microsatellites. Finer resolution population genomic studies as well as comparative genomics could potentially provide answers to these questions. These investigations could provide insight into the evolutionary implications of sympatric speciation in fungi.

Acknowledgments: We would like to thank P. Reeser and W. Sutton for providing support, assistance, and advice on the laboratory techniques and protocols used in this study. Z. Kamvar and J. Tabima provided assistance in Poppr troubleshooting and also helped to interpret figures and statistics used for population genetics inference. Funding from the Swiss Needle Cast Cooperative at Oregon State University, The Portland Garden Club, Cascade Mycological Society, and the US Forest Service is gratefully acknowledged. We would also like to acknowledge the Department of Botany and Plant Pathology as well as the Graduate School at Oregon State University for their generous contributions to student travel. We thank two anonymous reviewers for their constructive comments on an earlier draft.

Author Contributions: P.B. performed all sample processing, molecular work, and data analysis. J.S. and P.B. performed isolations and culture maintenance. P.B. originally wrote all text in this article with later additions and corrections by J.S. P.B. produced all figures and tables presented here.

Conflicts of Interest: The authors declare no conflict of interest.

\section{References}

1. Boyce, J.S. A needle-cast of Douglas Fir associated with Adelopus gaeumannii. Phytopathology 1940, 30, 649-659.

2. Hansen, E.M.; Stone, J.K.; Capitano, B.R.; Rosso, P.; Sutton, W.; Winton, L.; Kanaskie, A.; McWilliams, M.G. Incidence and impact of Swiss needle cast in forest plantations of Douglas-fir in coastal Oregon. Plant Dis. 2000, 84, 773-778. [CrossRef]

3. Manter, D.K.; Kavanagh, K.L.; Filip, G.M. Pseudothecia of Swiss needle cast fungus, Phaeocryptopus gaeumannii, physically block stomata of Douglas fir, reducing $\mathrm{CO}_{2}$ assimilation. New Phytol. 2000, 148, 481-491. [CrossRef]

4. Manter, D.K.; Winton, L.M.; Filip, G.M.; Stone, J.K. Assessment of Swiss needle cast disease: Temporal and spatial investigations of fungal colonization and symptom severity. J. Phytopathol. 2003, 151, 344-351. [CrossRef]

5. Oregon Department of Forestry. Swiss Needle Cast (SNC) Aerial Survey, 1996-2015. Avaliable online: http:/ / www.oregon.gov/odf (accessed on 28 September 2015).

6. Stone, J.K.; Capitano, B.R.; Kerrigan, J.L. The histopathology of Phaeocryptopus gaeumannii on Douglas-fir needles. Mycologia 2008, 100, 431-444. [CrossRef] [PubMed]

7. Winton, L.M.; Hansen, E.M.; Stone, J.K. Population structure suggests reproductively isolated lineages of Phaeocryptopus gaeumannii. Mycologia 2006, 98, 781-791. [CrossRef] [PubMed]

8. Winton, L.M. Phylogenetics, Population Genetics, Molecular Epidemiology, and Pathogenicity of the Douglas-Fir Swiss Needle Cast Pathogen Phaeocryptopus gaeumannii. Ph.D. Thesis, Oregon State University, Corvallis, OR, USA, 2001.

9. Winton, L.M.; Stone, J.K.; Hansen, E.M. Polymorphic microsatellite markers for the Douglas-fir pathogen Phaeocryptopus gaeumannii, causal agent of Swiss Needle Cast disease. Mol. Ecol. Notes 2007, 7, 1125-1128. [CrossRef]

10. R Core Team. R: A Language and Environment for Statistical Computing. R Foundation for Statistical Computing, Vienna, Austria. Available online: http:/ / www.R-project.org (accessed on 6 June 2013).

11. Peakall, R.; Smouse, P.E. Genalex 6: Genetic analysis in Excel. Population genetic software for teaching and research. Mol. Ecol. Notes 2006, 6, 288-295. [CrossRef] 
12. Peakall, R.; Smouse, P.E. Genalex 6.5: Genetic analysis in Excel. Population genetic software for teaching and research-An update. Bioinformatics 2012, 28, 2537-2539. [CrossRef] [PubMed]

13. Kamvar, Z.N.; Tabima, J.F.; Grünwald, N.J. Poppr: An R package for genetic analysis of populations with clonal, partially clonal, and/or sexual reproduction. PeerJ 2014, 2. [CrossRef] [PubMed]

14. Grünwald, N.J.; Hoheisel, G.A. Hierarchical analysis of diversity, selfing, and genetic differentiation in populations of the oomycete Aphanomyces euteiches. Phytopathology 2006, 96, 1134-1141. [CrossRef] [PubMed]

15. Stoddart, J.A.; Taylor, J.F. Genotypic diversity: Estimation and prediction in samples. Genetics 1988, 118, 705-711. [PubMed]

16. Simpson, E.H. Measurement of diversity. Nature 1949, 163. [CrossRef]

17. Nei, M. Estimation of average heterozygosity and genetic distance from a small number of individuals. Genetics 1978, 89, 583-590. [PubMed]

18. Grünwald, N.J.; Goodwin, S.B.; Milgroom, M.G.; Fry, W.E. Analysis of genotypic diversity data for populations of microorganisms. Phytopathology 2003, 93, 738-746. [CrossRef] [PubMed]

19. Excoffier, L.; Smouse, P.E.; Quattro, J.M. Analysis of molecular variance inferred from metric distances among DNA haplotypes: Application to human mitochondrial DNA restriction data. Genetics 1992, 131, 479-491. [PubMed]

20. Rambaut, A. FigTree Version 1.4.2. Available online: http://tree.bio.ed.ac.uk/software/figtree/ (accessed on 10 June 2014).

21. Rogers, J.S. Studies in Genetics. In Measures of Genetic Similarity and Genetic Distances; University of Texas Publication: Texas, TX, USA, 1972; pp. 145-153.

22. Manter, D.K.; Reeser, P.W.; Stone, J.K. A climate-based model for predicting geographic variation in Swiss needle cast severity in the Oregon Coast Range. Phytopathology 2005, 95, 1256-1265. [CrossRef] [PubMed]

23. Stone, J.K.; Hood, I.A.; Watt, M.S.; Kerrigan, J.L. Distribution of Swiss needle cast in New Zealand in relation to winter temperature. Australas. Plant Pathol. 2007, 36, 445-454. [CrossRef]

24. Stone, J.K.; Coop, L.B.; Manter, D.K. Predicting effects of climate change on Swiss needle cast disease severity in Pacific Northwest forests. Can. J. Plant Pathol. 2008, 30, 169-176. [CrossRef]

25. Watt, M.S.; Stone, J.K.; Hood, I.A.; Palmer, D.J. Predicting the severity of Swiss needle cast on Douglas-fir under current and future climate in New Zealand. For. Ecol. Manag. 2010, 260, 2232-2240. [CrossRef]

26. Watt, M.S.; Stone, J.K.; Hood, I.A.; Manning, L.K. Using a climatic niche model to predict the direct and indirect impacts of climate change on the distribution of Douglas-fir in New Zealand. Glob. Chang. Biol. 2011, 17, 3608-3619. [CrossRef]

27. Balloux, F.; Lugon Moulin, N. The estimation of population differentiation with microsatellite markers. Mol. Ecol. 2002, 11, 155-165. [CrossRef] [PubMed]

(C) 2016 by the authors; licensee MDPI, Basel, Switzerland. This article is an open access article distributed under the terms and conditions of the Creative Commons by Attribution (CC-BY) license (http://creativecommons.org/licenses/by/4.0/). 